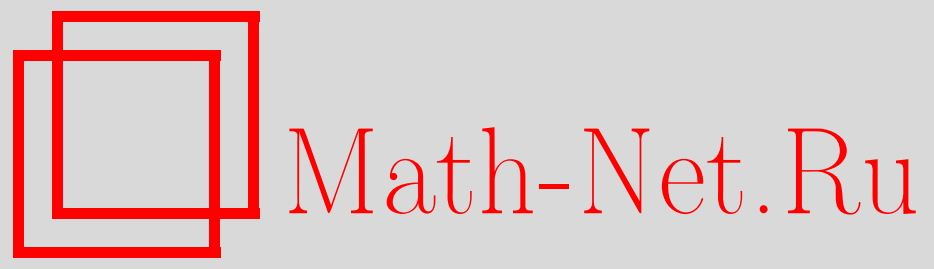

В. И. Рабанович, Ю. С. Самойленко, Когда сумма идемпотентов или проекторов кратна единице, Функи. анализ и его прил., 2000, том 34, выпуск 4, 91-93

DOI: https://doi.org/10.4213/faa333

Использование Общероссийского математического портала MathNet.Ru подразумевает, что вы прочитали и согласны с пользовательским соглашением

http://www. mathnet.ru/rus/agreement

Параметры загрузки:

IP: 54.80 .97 .219

26 апреля 2023 г., $17: 10: 25$

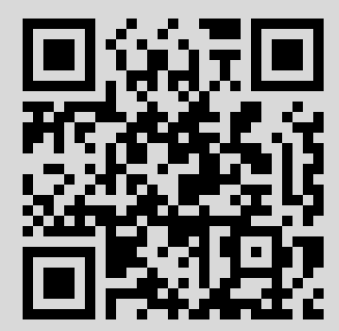


УДК 517.98

\title{
Когда сумма идемпотентов или проекторов кратна единице*
}

\author{
(С) 2000. В. И. РАБАНОВИЧ, Ю. С. САМОЙЛЕНКО
}

Пусть $H$ - сепарабельное гильбертово пространство (конечномерное или счетномерное), $L(H)$ - пространство ограниченных операторов в $H$ и $I$ - единичный оператор в $H$. Операторы $Q$, такие, что $Q=Q^{2}$, называются идемпотентами в $L(H)$, а операторы $P$, для которых $P=P^{2}=P^{*},-$ проекторами. В целом ряде работ (см., например, [1-10] и библиографию, приведенную в них) исследуется множество операторов, которые могут быть представлены в виде линейной комбинации конечного числа идемпотентов или проекторов. В настоящей работе изучаются множества $\Lambda_{1} \subset \ldots \subset \Lambda_{n}=\{\lambda \in \mathbb{C} \mid$ существуют идемпотенты $Q_{1}, \ldots, Q_{n}$, такие, что $\left.\sum_{k=1}^{n} Q_{k}=\lambda \cdot I\right\} \subset \Lambda_{n+1} \subset \ldots \subset \Lambda_{\infty}=\bigcup_{n=1}^{\infty} \Lambda_{n}$ $\subset \mathbb{C}$ и множества $\Sigma_{1} \subset \ldots \subset \Sigma_{n}=\left\{\alpha \in \mathbb{R} \mid\right.$ существуют $n$ проекторов $P_{1}, \ldots, P_{n}$, таких, что $\left.\sum_{k=1}^{n} P_{k}=\alpha \cdot I\right\} \subset \Sigma_{n+1} \subset \ldots \subset \Sigma_{\infty}=\bigcup_{n=1}^{\infty} \Sigma_{n} \subset \mathbb{R}$. Результаты заметки получены при изучении представлений алгебр $\mathbb{C}\left\langle q_{1}, \ldots, q_{n}\right| q_{k}^{2}=q_{k}$, $\left.\left[\sum_{j=1}^{n} q_{j}, q_{k}\right]=0, k=1, \ldots, n\right\rangle$ и *-алгебр $\mathbb{C}\left\langle p_{1}, \ldots, p_{n}\right| p_{k}^{2}=p_{k}^{*}=p_{k},\left[\sum_{j=1}^{n} p_{j}, p_{k}\right]$ $=0, k=1, \ldots, n\rangle$ и их применения к задачам теории операторов и операторных алгебр (см., например, обзор [6]).

1. Предварительная информация. Так как для любого идемпотента $Q_{k}$ оператор $I-Q_{k}$ также является идемпотентом, а для любого проектора $P_{k}$ оператор $I-P_{k}-$ проектор, то из того, что $\lambda \in \Lambda_{n}$, следует включение $n-\lambda \in \Lambda_{n}$. Аналогично включение $\alpha \in \Sigma_{n}$ приводит к включению $n-\alpha \in \Sigma_{n}$. Имеет место

ПРЕДЛОЖЕНИЕ 1. $\Sigma_{n} \subset\left\{0,1,1+\frac{1}{n-1},\left[1+\frac{1}{n-2}, n-1-\frac{1}{n-2}\right], n-1-\frac{1}{n-1}, n-1, n\right\}$.

ПримеР 1. Для любого $n \geqslant 2$ существуют (строятся) $n$ проекторов $P_{1}, \ldots, P_{n}$ в конечномерном пространстве $H(\operatorname{dim} H=n-1)$, таких, что $\sum_{k=1}^{n} P_{k}=\left(1+\frac{1}{n-1}\right)$. $I$. Для всех $i \neq j$ проекторы $P_{i}$ и $P_{j}$ не коммутируют, и семейство проекторов $P_{1}, \ldots, P_{n}$ неприводимо. Таким образом, $\Sigma_{n} \ni 1+\frac{1}{k-1}$ при всех $k \leqslant n$.

Пример 2. Для любого рационального $r, 1>r=m / n>0$, существуют (строятся) проекторы $P_{1}, \ldots, P_{n+m}$ в $H(\operatorname{dim} H=n)$, такие, что $P_{1}+\cdots$ $+P_{n+m}=(1+r) I$. Семейство $P_{1}, \ldots, P_{n+m}$ неприводимо. Таким образом, $\Sigma_{\infty}$ содержит все рациональные $r \geqslant 1$.

Примеры 1 и 2 можно построить при помощи теоремы 1 из [2].

ЗАмечАниЕ 1. Если $H$ конечномерно, то ненулевые решения уравнения $\sum_{1}^{n} Q_{k}=\lambda I$ могут существовать лишь при вещественных рациональных $\lambda=$ $\left(\sum_{k=1}^{n} \operatorname{Tr}\left(Q_{k}\right)\right) / \operatorname{dim} H \geqslant 1$, так как $\sum_{k=1}^{n} \operatorname{Tr}\left(Q_{k}\right) \in \mathbb{N}$. Таким образом, множество тех $\lambda \in \Lambda_{\infty}$, для которых существуют ненулевые идемпотенты с суммой $\lambda I$, в случае конечномерного пространства $H$ совпадает с множеством рациональных $\lambda \geqslant 1$. Это следует также из результатов статьи [3].

\section{2. $\Lambda_{n}$ и $\Sigma_{n}$ при $n \leqslant 4$.}

* Исследования второго автора частично поддержаны грантом CRDF UM1-311. 
TeOpema 1. $\Sigma_{1}=\Lambda_{1}=\{0,1\} \subset \Sigma_{2}=\Lambda_{2}=\{0,1,2\} \subset \Sigma_{3}=\Lambda_{3}=\left\{0,1, \frac{3}{2}, 2,3\right\}$ $\subset \Sigma_{4}=\Lambda_{4}=\left\{0,1,2 \pm \frac{1}{k}(k \geqslant 2), 2,2 \pm \frac{2}{2 k+1}(k \in \mathbb{N}), 3,4\right\}$.

Описание множеств $\Sigma_{n}$ и $\Lambda_{n}, n \leqslant 4$, известно (см., например, [4, 6, 8, 9] и др.). Совпадение множеств $\Sigma_{n}$ и $\Lambda_{n}$ при $n \leqslant 4$ не случайно. Оказывается, что при $n=4(\lambda \neq 2)$ для любого представления $\pi$ алгебры $Q_{4, \lambda}=\mathbb{C}\left\langle q_{1}, \ldots, q_{4}\right|$ $\left.q_{k}^{2}=q_{k}(k=1, \ldots, 4), \sum_{1}^{4} q_{k}=\lambda e\right\rangle$ в $L(H)$ в $H$ существует такое эквивалентное скалярное произведение, что $\pi$ в новом скалярном произведении становится *-представлением $*$-алгебры $\mathscr{P}_{4, \lambda}=\mathbb{C}\left\langle p_{1}, \ldots, p_{4}\right| p_{k}^{2}=p_{k}=p^{*}(k=1, \ldots, 4)$, $\left.\sum_{1}^{4} p_{k}=\lambda e\right\rangle$. Все неприводимые $*$-представления алгебры $\mathscr{P}_{4, \lambda}(\lambda=\alpha>0)$ конечномерны. Описание неприводимых $*$-представлений $*$-алгебр $\mathscr{P}_{4, \alpha}$ см. в [5, 9-11].

ЗАмЕЧАНИЕ 2 . В конечномерном векторном пространстве $H$ представление единичного оператора в виде суммы $n$ идемпотентов является ортогональным разложением единицы, т. е. обладает свойством $Q_{i} Q_{j}=Q_{j} Q_{i}=0, i \neq j$ (см., например, [12]). Как следует из доказательства теоремы 1 , при $n \leqslant 4$ это верно и когда $H$ бесконечномерно. Конструкция операторов из теоремы 2 ниже показывает, что при $n \geqslant 5$ не каждое представление единичного оператора в виде суммы $n$ идемпотентов является ортогональным.

\section{3. $\Lambda_{n}(n=5, \ldots, \infty)$.}

TEOPEMA 2. $\Lambda_{5}=\Lambda_{\infty}=\mathbb{C}$.

ДокАЗАТЕЛЬСтво. Утверждение теоремы непосредственно следует из [1], где доказано, что любой ограниченый оператор в $H$ есть сумма пяти идемпотентов. Для любого $\lambda \in \mathbb{C}$ мы приведем непосредственную конструкцию пяти идемпотентов $Q_{i}=Q_{i}^{2} \in L(H)(i=1, \ldots, 5)$, которые в сумме дают скалярный оператор $\lambda I$. Пусть $H=l_{2} \oplus l_{2} \oplus l_{2}$ и $\mathscr{I}-$ тождественный оператор в $l_{2}$. Определим следующие операторы из $L(H)$ :

$$
\begin{aligned}
& Q_{1}=\left(\begin{array}{ccc}
a \mathscr{I} & 3 a \mathscr{I} & \frac{1}{3}(4 \lambda-7) \cdot \mathscr{I} \\
a \mathscr{I} & 3 a \mathscr{I} & \frac{1}{3}(4 \lambda-7) \cdot \mathscr{I} \\
a \mathscr{I} & 3 a \mathscr{I} & \frac{1}{3}(4 \lambda-7) \cdot \mathscr{I}
\end{array}\right), \quad Q_{2}=\left(\begin{array}{ccc}
a \mathscr{I} & -3 a \mathscr{I} & \frac{1}{3}(4 \lambda-7) \cdot \mathscr{I} \\
-a \mathscr{I} & 3 a \mathscr{I} & -\frac{1}{3}(4 \lambda-7) \cdot \mathscr{I} \\
a \mathscr{I} & -3 a \mathscr{I} & \frac{1}{3}(4 \lambda-7) \cdot \mathscr{I}
\end{array}\right), \\
& Q_{3}=\left(\begin{array}{ccc}
4 a \mathscr{I} & 0 & -\frac{2}{3}(4 \lambda-7) \cdot \mathscr{I} \\
0 & 0 & 0 \\
-2 a \mathscr{I} & 0 & \frac{1}{3}(4 \lambda-7) \cdot \mathscr{I}
\end{array}\right), \quad Q_{4}=\left(\begin{array}{ccc}
2 b \mathscr{I} & 0 & (7-3 \lambda) b \cdot S_{1}^{*} \\
0 & 2 b \mathscr{I} & (7-3 \lambda) b \cdot S_{2}^{*} \\
S_{1} & S_{2} & \frac{1}{2}(7-3 \lambda) \cdot \mathscr{I}
\end{array}\right), \\
& Q_{5}=\left(\begin{array}{ccc}
2 b \mathscr{I} & 0 & -(7-3 \lambda) b \cdot S_{1}^{*} \\
0 & 2 b \mathscr{I} & -(7-3 \lambda) b \cdot S_{2}^{*} \\
-S_{1} & -S_{2} & \frac{1}{2}(7-3 \lambda) \cdot \mathscr{I}
\end{array}\right),
\end{aligned}
$$

где $a=\frac{1}{6}(5-2 \lambda), b=\frac{1}{4}(3 \lambda-5), S_{1}^{*}\left(x_{0}, x_{1}, \ldots\right)=\left(x_{0}, x_{2}, x_{4}, \ldots\right), S_{2}^{*}\left(x_{0}, x_{1}, \ldots\right)=$ $\left(x_{1}, x_{3}, x_{5}, \ldots\right), S_{1}\left(x_{0}, x_{1}, \ldots\right)=\left(x_{0}, 0, x_{1}, 0, x_{2}, \ldots\right)$ и $S_{2}\left(x_{0}, x_{1}, \ldots\right)=\left(0, x_{0}, 0\right.$, $\left.x_{1}, 0, x_{2}, \ldots\right)$. Операторы $S_{i}, S_{i}^{*}$ связаны соотношениями $S_{1}^{*} S_{2}=0$ и $S_{1}^{*} S_{1}=I=$ $S_{2}^{*} S_{2}=S_{1} S_{1}^{*}+S_{2} S_{2}^{*}$.

Непосредственно проверяется, что

$$
Q_{1}+Q_{2}+Q_{3}+Q_{4}+Q_{5}=\lambda \cdot I .
$$

Эта конструкция является обобщением примера, приведенного в [7], пяти идемпотентов, сумма которых равна нулю. 
ЗАмЕчАНИЕ 3. Из приведенных в доказательстве соотношений между $S_{i}$ следует, что любое представление алгебры Кунца $\mathscr{Q}_{2}$ дает подходящую пару операторов $S_{1}, S_{2}[13]$.

\section{4. $\Sigma_{n}(n \geqslant 6, n=\infty)$.}

TЕОРемА 3. Если $n \geqslant 6$, то $\Sigma_{n} \supset\left\{0,1,1+\frac{k}{k(n-3)+2}(k \in \mathbb{N}),\left[1+\frac{1}{n-3}, n-1-\right.\right.$ $\left.\left.\frac{1}{n-3}\right], n-1-\frac{k}{k(n-3)+2}(k \in \mathbb{N}), n-1, n\right\}$.

Ее доказательство основано на следующих трех леммах:

ЛЕммА 1. $\Sigma_{n} \supset\left\{1+\frac{k}{k(n-3)+2}, k \in \mathbb{N}\right\}$ для любого $n \geqslant 6$.

ЛЕММА 2. $\Sigma_{n} \supset[2, n-2]$ Әля $n \geqslant 6$.

ЛЕммА 3. $\Sigma_{n} \supset\left\{\left[1+\frac{1}{n-3}, 2\right]\right\}$ для $n \geqslant 6$.

Доказательство лемм конструктивно.

СлеДСТВИЕ 1. $\Sigma_{\infty}=\{0,[1, \infty)\}$.

ЗАмЕчАНиЕ 4. Следствие 1 можно получить также, применяя теорему 4.12 из [6].

Авторы благодарны профессорам В. С. Шульману и Торстену Эрхардту за полезные обсуждения изложенных вопросов.

\section{ЛИТЕРАТУРА}

1. Pearcy C., Topping D. M. Michigan Math. J., 14, 453-465 (1967). 2. Fillmore P. A. J. Funct. Anal., 4, 146-152 (1969). 3. Hartwing R. E., Putcha M. S. Linear Multilinear Algebra, 26, 279-286 (1990). 4. Wang J.-H. Ph. D. Dissertation, National Chiao Tung Univ., Hsinchu, Taiwan, 1991. 5. Беспалов Ю. Н. Укр. матем. ж., 44, 309-317 (1992). 6. Wu P. $Y$. In: Functional Analysis and Operator Theory, Banach Center Publ., vol. 30, Warszawa, 1994, 337-361. 7. Bart H., Ehrhardt T., Silbermann B. Integral Equations Operator Theory, 19, 123-134 (1994). 8. Ehrhardt T. (unpublished). Chemnitz, 1995. 9. Galinsky D. V., Muratov M. A. In: Spectral Evolutionary Problems, no. 8, Simferopol, Tavria, 1998, pp. 1522. 10. Ostrouskii $V$., Samoilenko Yu. Introduction to the Theory of Representations of Finitely Presented $*$-Algebras. 1. Representations by Bounded Operators. Gordon and Breach, London, 1999. 11. Городний М. Ф., Подколзин Г. Б. В кн.: Спектральная теория операторов и бесконечномерный анализ, ИМ АН УССР, Киев, 1984, с. 66-76. 12. Глазман И. М., Любич Ю. И. Конечномерный линейный анализ в задачах. Наука, M., 1969. 13. Cuntz J. Comm. Math. Phys., 57, 173-185 (1977).

Институт математики НАНУ,

Киев, Украина

Поступило в редакцию 9 апреля 1999 г. 